\title{
ORL1 et la nociceptine: l'amorce d'une nouvelle famille de récepteurs et de ligands endogènes associés aux systèmes des opiacés?
}

L'introduction de la génétique moléculaire dans l'étude des récepteurs peptidiques a contribué, lors du clonage de l'ADNc de récepteurs, à la découverte de nombreux sous-types de récepteurs d'un même ligand mais également à la découverte de récepteurs orphelins de ligands endogènes inconnus. En 1994, un premier travail coopératif (Toulouse, France, Bruxelles, Belgique) dirigé par JeanClaude Meunier à Toulouse avait permis de décrire le clonage d'un nouveau membre de la famille des récepteurs des opiacés, le récepteur ORL1 (pour opioid receptor-like 1) sans que l'on connût son ligand endogène [1]. Ce travail faisait suite au clonage des ADNc et à la description en 1992 et 1993 des récepteurs des opiacés classiques $\mu, \delta$ et $\kappa$, protéines monomériques à la structure de récepteurs à sept domaines transmembranaires couplés à une protéine $\mathrm{G}$ [2]. Le premier de ces récepteurs dont l'ADNc a été cloné, le récepteur $\delta$, présentait des analogies avec les récepteurs de la somatostatine, un peptide initialement décrit comme hormone hypothalamique inhibitrice de la libération de l'hormone de croissance par les cellules somatotropes de l'adénohypophyse. Cette analogie entre les deux types de récepteurs s'avérait intéressante dans la mesure où des agonistes du récepteur de la somatostatine possèdent des propriétés analgésiques. Meunier et al. avaient utilisé une technique d'amplification sélective par PCR d'ADN génomique humain avec des oligonucléotides codant pour des régions très conservées des récepteurs des opiacés $\delta$ et de la somatostatine pour synthétiser une sonde d'ADN de 761 paires de base. Cette sonde leur permit de cribler une banque d'ADNc constituée à

d'identifier un clone d'ADNc codant pour une protéine de 370 acides aminés qu'ils appelèrent ORL1 [1].

La séquence de ce récepteur est très bien conservée chez les mammifères avec, par exemple, entre la souris et l'homme $87 \%$ d'identité en ce qui concerne les nucléotides, un pourcentage qui s'élève à $99 \%$ si l'on considère la séquence des acides aminés. La structure de ce récepteur est très proche de celle des trois récepteurs des opiacés dont il partage beaucoup de caractères structuraux, principalement dans les domaines transmembranaires $(67 \%$ d'analogie) et dans la boucle cytoplasmique du fragment C-terminal (75\% d'analogie). Dans des cellules CHO transfectées avec une construction codant pour ORL1, l'activation de ce récepteur par l'étorphine, ligand universel des récepteurs des opiacés, entraîne une inhibition de l'adénylyl cyclase, un effet inversé par la diprénorphine, antagoniste universel de cette même famille. Mais l'étude pharmacologique réalisée à l'aide de substances opiacées endogènes ou synthétiques sur ces cellules transfectées avait permis de montrer que ORL1 n'est pas un récepteur des opiacés classique et que son profil pharmacologique restait à déterminer. L'étude par hybridation in situ réalisée par plusieurs équipes avec des transcrits ARN de type ORL1 a montré que la distribution de ce récepteur était très large, tant à la périphérie (intestin, canal déférent et rate) que dans le système nerveux central du rat et de la souris, mais localisée dans des structures bien délimitées (hypothalamus, aires limbiques, pont et moelle épinière dorsale et ventrale), suggérant que ORL1 pourrait être associé à plusieurs fonctions.
D'après la séquence des acides aminés déduite de celle du gène, il était probable que le ligand endogène ressemblât à une dynorphine A, un hepta-décapeptide agoniste endogène du récepteur des opiacés $\kappa$. Ainsi, en utilisant une procédure d'extraction identique à celle décrite lors de l'isolement de la dynorphine et en mesurant comparativement l'activité d'extraits peptidiques de cerveau de rat sur l'activité adénylyl cyclase de cellules CHO recombinées exprimant le gène ORL1 et de cellules CHO non recombinées, un peptide exerçant un effet inhibiteur puissant sur l'enzyme a été isolé et séquencé [3]. Il s'agit d'un peptide de dix-sept résidus, proche dans sa structure de la dynorphine A (six acides aminés conservés dans la séquence). Le produit purifié de même que le peptide synthétique inhibent à des concentrations nanomolaires l'activité adénylyl cyclase de cellules recombinantes CHO transfectées avec une construction codant pour ORL1. Les auteurs de ce travail ont également montré que le neuropeptide naturel existe sous la forme d'un précurseur peptidique beaucoup plus grand, qui possède des motifs d'excision protéolytiques. Le peptide synthétique s'est avéré actif in vivo mais, curieusement, dans un sens opposé à celui attendu pour une inhibition de l'adénylyl cyclase: injecté par voie intracérébroventriculaire chez la souris, il diminue les temps de latence de redressement et du saut dans le test de la plaque chaude, un test couramment utilisé pour mesurer l'activité analgésique de molécules qui ont une action opposée et augmentent ces temps de latence. Vingt minutes après l'injection du peptide, les animaux deviennent hyper-réactifs à la stimulation nociceptive thermique de 
la plaque chaude: ils se redressent sur leurs pattes postérieures puis sautent hors de la plaque chaude avec des temps de latence plus courts que les animaux témoins. En outre, l'injection intracérébroventriculaire répétée d'un oligonucléotide antisens de l'ARNm du récepteur ORL1 a eu l'effet opposé à celui du peptide endogène: lorsque la synthèse d'ORL1 est diminué, les temps de latence mesurés par ce même test sont augmentés. Compte tenu de l'effet d'hyper-réactivité à la stimulation nociceptive thermique (plaque chaude) relayé par ce peptide, les auteurs l'ont appelé "nociceptine».

Avec la mise en évidence d'une nouvelle famille de récepteurs et de ligands endogènes de type opiacé dans le système nerveux, une voie vient d'être ouverte dans la compréhension du fonctionnement de ces systèmes qui pourrait avoir des conséquences pharmacologiques importantes. Il reste à comprendre les rai- sons de cette opposition entre les effets cellulaires de type morphinique du peptide (inhibition de l'adénylyl cyclase), et ses effets pro-nociceptifs, de type antimorphinique, lorsqu'il est testé in vivo. La mesure de cette activité in vivo avec des tests nociceptifs autres que la plaque chaude devrait permettre de comprendre cette opposition et de pouvoir ainsi vérifier si la nociceptine est la bien-nommée ! Comme un fait exprès, ce peptide a reçu un autre nom, l'orphanine $\mathrm{FQ}$, nom qui reflète sans aucun doute les incertitudes sur la fonction physiologique exacte de ce peptide in vivo [4]. En effet, dans une étude simultanée réalisée par une équipe suisse, un ligand du récepteur ORL1, appelé aussi récepteur LC132 a été isolé, séquencé et correspond tout à fait à la nociceptine. De façon surprenante dans le test de la plaque chaude, le peptide n'a pas d'effet nociceptif mais exerce plutôt une action antinociceptive lorsqu'il est injecté à fortes concentrations à des souris. Cet effet a été mis en relation avec la diminution de l'activité locomotrice et du tonus musculaire de ces souris. En revanche, un effet hyperalgésique important est observé dans le test de tail-flick, ce qui est en accord avec un effet nociceptif prépondérant du peptide.

B.C.

B.A.

1. Mollereau C, Parmentier M, Mailleux P, et al. ORL1, a novel member of the opioid receptor family. Cloning, functional expression and localization. FEBS Lett 1994; 341 : 33-8.

2. Noel F, Iourgenko V, Pouille Y, Hanoune J. Les mécanismes d'action moléculaire des opiacés. médecine/sciences $1994 ; 10$ : 1116-26.

3. Meunier JC, Mollereau C, Toll L, et al. (14 auteurs). Isolation and structure of the endogenous agonist of opioid receptor-like ORL1 receptor. Nature $1995 ; 377$ : 532-5.

4. Reinscheid RK, Nothacker HP, Bourson A, Ardati A, Henningsen RA, Bunzow JR, Grandy DK, Langen H, Monsma FJ, Civelli O. Orphanin FQ: a neuropeptide that activates an opioidlike $\mathrm{G}$ protein-coupled receptor. Science 1995; 270: 792-4.

\section{BRÈVES}

Obésité et cerveau : de nouveaux indices ! Depuis la découverte du "gène de l'obésité ", dont la mutation induit une obésité chez la souris $(\mathrm{m} / \mathrm{s}$ $n^{\circ} 12$, vol. 10, p. 1337) [1], un gène homologue a été identifié chez le rat et l'homme. Le gène $o b$, qui ne s'exprime que dans le tissu adipeux, code pour une protéine de $16 \mathrm{kDa}(\mathrm{OB})$, synthétisée et sécrétée par les cellules adipeuses et retrouvée dans le plasma. Il est aujourd'hui admis que le pouvoir amaigrissant de cette protéine, chez la souris tout au moins, est dû à sa double fonction, inhibitrice sur la prise alimentaire et activatrice sur la consommation d'énergie, conduisant ainsi à l'augmentation de la mobilisation des graisses corporelles. La question essentielle, dont la réponse constituerait une avancée importante dans la compréhension des mécanismes de l'obésité, concerne le site d'action de la protéine $O B$. Des expériences préliminaires avaient montré que l'administration directe de la protéine $\mathrm{OB}$ dans le ventricule cérébral latéral modifiait la prise alimentaire et l'équilibre énergétique chez des souris $o b / o b$, suggérant ainsi que une ou plu- sieurs régions du cerveau étaient les cibles de OB. En outre, la participation d'un neuropeptide " antiobésité » comme le neuropeptide Y (NPY) était fortement pressentie au vu de ses capacités régulatrices sur la prise alimentaire, la thermogenèse et les sécrétions d'insuline et de corticostérone [2, 3]. L'article de T.W Stephens et al. (Lilly Research Laboratories) [4] confirme ces hypothèses puisqu'il vient de montrer que : 1 ) un traitement de 30 jours de souris $o b / o b$ par la protéine OB recombinée humaine conduit à une normalisation de la prise alimentaire et du poids des souris. Cette modification s'accompagne d'une diminution des ARNm du NPY dans l'hypothalamus, suggérant une baisse de la synthèse de NPY dans ce tissu. Cette diminution n'a pas été observée chez la souris obèse $d b / d b$ chez laquelle l'effet amaigrissant de la protéine OB n'est pas observé ; 2) un effet inhibiteur direct de OB sur la sécrétion de NPY par l'hypothalamus a été mis en évidence sur des hypothalamus isolés et perfusés in vitro; 3 ) chez la souris $o b / o b$, l'injection intraventriculaire de $\mathrm{OB}$ recombinée de souris est plus efficace sur la baisse de la prise alimentaire qu'une administration sous-cutanée ; 4) des récepteurs spécifiques et de forte affinité pour la protéine OB de souris ont été identifiés dans des membranes d'hypothalamus de rat. L'ensemble de ces observations est tout à fait en faveur d'une action hypothalamique de $\mathrm{OB}$ et d'un mécanisme régulateur « amaigrissant » mettant en jeu le NPY. Il est cependant intéressant de noter que d'autres neuropeptides ayant des effets régulateurs sur la prise alimentaire et se liant à des récepteurs spécifiques au niveau de l'hypothalamus (cholécystokinine, galanine, somatostatine...) pourraient être également des candidats potentiels pour relayer l'action de la protéine $\mathrm{OB}$ au niveau du système nerveux central.

[1. Zhang Y, et al. Nature 1994 ; 372: 425-32.]

[2. Andersen A, et al. médecine/ sciences $1987 ; 3: 293-5$.]

[3. Castan I, et al. médecine/sciences 1994 ; 10 : 196-201.]

[4. Stephens TW et al. Nature 1995 ; $377: 530-2$.
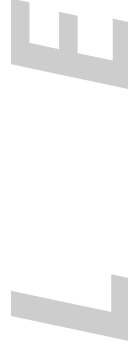

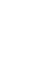

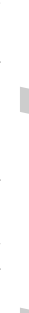

